\title{
Reconocimiento de metáforas de literatura en niños escolares
}

\section{Metaphor recognition in literature by schoolchildren}

\author{
Patricia Pineda 1
}

Citation/ Para citar este Artículo: Pineda, P. (2018). Reconocimiento de metáforas de literatura en niños escolares. Colomb. appl. linguist.j., 20(1), pp. 25-34.

Received: 03-Apr.-2017 / Accepted: 26-Jun.-2017

DOI: https://doi.org/10.14483/22487085.11818

\section{Resumen}

Dada la necesidad de avanzar en la comprensión del reconocimiento de usos metafóricos en niños ${ }^{2}$ escolares desde una perspectiva psicolingüística del lenguaje en uso que supere la mirada de la psicología del desarrollo, este artículo presenta los resultados de un estudio de reconocimiento de metáforas en textos de literatura infantil. Se tiene como premisa que la metáfora lingüística es un fenómeno de uso del lenguaje y que el análisis de su procesamiento supone considerar los procesos de comprensión textual. El material lingüístico fue tomado de la literatura infantil y se utilizó una tarea de subrayado. Se optó por un diseño ANOVA con dos factores inter-sujeto (comprensión lectora y grado escolar). Los resultados indican avances en el reconocimiento metafórico con el grado escolar y con el nivel de comprensión lectora y una significativa influencia de esta última, sobrepasando en algunos casos a la del grado escolar; así como diferencias importantes en desempeños de niños con el mismo nivel de comprensión lectora. Como conclusión se plantea la relevancia de la comprensión lectora y la importancia de identificar el rol de los diferentes aspectos de ésta en el reconocimiento metafórico.

Palabras clave: comprensión lectora, consciencia metaléxica, desarrollo metalingüístico, literatura infantil, reconocimiento metafórico.

\begin{abstract}
Given the need to advance in the understanding of the recognition of metaphorical uses by schoolchildren from a psycholinguistic perspective of language in use that goes beyond the perspective of developmental psychology, this article presents the results of a study about metaphor recognition by children. The premise is that the linguistic metaphor is a phenomenon of language use and that the analysis of its processing has to take into account the processes of textual comprehension. The linguistic material was taken from children's literature and participants were asked to underline metaphors. Statistical analysis was made using ANOVA with two inter-subject factors (reading comprehension and school grade). The results indicate advances in metaphorical recognition with school grade and level of reading comprehension and a significant influence of the latter, exceeding in some cases that of the school grade; as well as important differences in performance of children with the same level of reading comprehension. These findings provide evidence about the relevance of reading comprehension but also stress the importance to better identify the role of the different sub-skills involved in reading comprehension in metaphor recognition.
\end{abstract}

Keywords: children literature, metalexical awareness, metalinguistic development, metaphor recognition, reading comprehension.

1 Investigadora independiente, Colombia. p.pinedaz@yahoo.com

2 Se utilizará este término para referirse al conjunto de participantes: niños y niñas. 


\section{Introducción}

La exploración del reconocimiento explícito de metáforas es un reto dada la limitada investigación al respecto, a pesar de la importancia que reviste para el desarrollo metalingüístico y la comprensión y producción del lenguaje. Existen algunos estudios con adultos (Graesser, Mio, E Millis, 1988; Steen, 1994; Steen, 2004) y sorpresivamente aún menos con niños (Cameron, 2003; De Groot, Kaplan, Rosenblatt, \& Winner, 1995).

La teoría conceptual (Lakoff $\mathcal{E}$ Johnson, 1980) marcó un giro significativo en la investigación en metáfora, que pasó de ser pensada como una peculiaridad lingüística a tener un lugar importante en la cognición. Este cambio de concepción ha facilitado un mayor desarrollo de estudios y escuelas que, no obstante sus diferencias, parecen estar de acuerdo con la definición básica de metáfora acuñada por los gestores de la teoría conceptual (Lakoff $\mathcal{E}$ Johnson, 1980, p. 41) que "la esencia de la metáfora es entender y experimentar una cosa en términos de otra". Dicha comprensión y experimentación implicaría una asociación ('mapping', en inglés) entre dos dominios conceptuales diferentes que están puestos en juego en una metáfora. Por ejemplo y siguiendo a Lakoff y Johnson (1980, p. 40), en la expresión lingüística sus críticas dieron justo en el blanco, los autores postulan que se trata de los dominios conceptuales discusión y guerra.

La asociación o proceso de conexión entre dos dominios conceptuales indicado como necesario para procesar metáforas ha sido puesto en cuestión (Bowdle \& Gentner, 2005; Gentner \& Bowdle, 2001, 2008; Glucksberg, 2008; Steen, 2008, 2011). Particularmente Steen $(2008,2011)$ argumenta que los resultados de investigaciones en procesamiento de metáforas indicarían que la mayoría de estas no son procesadas metafóricamente, es decir pasando por un proceso de conexión o asociación entre dominios conceptuales. Steen (2011) señala la necesidad de incluir en el análisis la dimensión comunicativa y concretamente el aspecto de si la metáfora es o no usada o recibida deliberadamente, de tal manera que genere un cambio de perspectiva que implique considerar una cosa en términos de otra.
Es necesario tener en cuenta que es fundamental diferenciar entre el análisis desde la aproximación semiótica o simbólica que hace el analista del lenguaje y desde la del procesamiento mental que hacen los hablantes de una lengua, cuando usan, comprenden o como los participantes de este estudio, reconocen metáforas.

Con base en estas dos aproximaciones se adoptó la definición de metáforas lingüísticas propuesta por Cameron (1996, p. 52) como "partes del lenguaje que son identificadas por el analista como metáforas de acuerdo con criterios establecidos y que se considera que tienen el potencial de ser procesadas como metáforas".

Ahora bien, que una metáfora sea procesada como metáfora no es una condición para su comprensión. La investigación actual en psicolingüística sugiere que el proceso de comprensión de metáforas se da en pocos segundos, lo que implica que una metáfora puede ser perfectamente comprendida sin que haya consciencia de que se trata de una metáfora (Gibbs, 1993, 1994; Steen, 1994, 2011). En la comprensión, por tanto, no tiene lugar necesariamente un proceso consciente de asociación o de conexión entre dos dominios conceptuales, en donde se experimente una potencial diferencia entre el significado sugerido en la metáfora y el significado más básico del o de los términos utilizados para decir una cosa en términos de otra. Lo anterior quiere decir que muchas metáforas pueden pasar desapercibidas para un usuario común del lenguaje, y por ende no serían reconocidas como metáforas, aunque sean adecuadamente comprendidas. Reconocimiento e interpretación de metáforas son entonces dos operaciones mentales diferentes e independientes que pueden o no tener lugar conjuntamente.

El reconocimiento de metáforas es un proceso psicológico (mental) en donde el usuario de una lengua se percata en menor o mayor medida dado que la consciencia no es necesariamente una cuestión de todo o nada-, de las diferencias entre el significado básico y el significado contextual de un término o términos usados metafóricamente. Asimismo, puede también atribuir una específica intencionalidad comunicativa (otorgar al usuario 
del enunciado el propósito de presentar una cosa en términos de otra), y en algunos casos hasta explicitar el tipo de uso del lenguaje, metáfora, de que se trata (Steen, 1994, 2004).

Llegados a este punto es importante distinguir entre lo que Steen (2004) nombra como reconocimiento 'espontáneo' de metáforas y el reconocimiento generado o provocado por una tarea o pedido de un tercero. En este último es esperable que el lector se encuentre más focalizado en la búsqueda de metáforas.

\section{Estudios previos en reconocimiento de metáforas}

Los resultados del primer estudio de reconocimiento de metáforas realizado hace dos décadas (De Groot, et al., 1995) con niños de dos grupos de edad y con metáforas que fueron presentadas al final de una corta historia, muestran que el grupo de niños de siete años reconoció significativamente más metáforas que el de seis. En un estudio más reciente (Cameron, 2003), en donde participaron dos niñas de nueve y diez años se encontró que las metáforas nominales (en donde un nombre o sustantivo es usado metafóricamente) presentes en dos fragmentos de texto de ciencias naturales que las niñas debieron leer y discutir, tuvieron más posibilidades de ser notadas que aquellas en donde la parte usada metafóricamente corresponde a un verbo.

Según Olson (1988) reconocer una metáfora como metáfora demanda que el niño tenga el concepto de metáfora, de tal forma que pueda distinguir entre el significado del término o términos usados metafóricamente y el significado más básico de dicho(s) término(s) e identificar el sentido metafórico sugerido por el contexto. Poder establecer esa diferencia entre los diferentes significados de un término implica tener el grado de desarrollo cognitivo y lingüístico que permita diferenciar entre un evento (físico o lingüístico) y la representación de ese evento (Olson, 1988) y, en esa medida, aceptar que un enunciado pueda tener diferentes interpretaciones. La investigación en el desarrollo de esta diferenciación entre lo que se dice y lo implicado (Torrance y Olson,
1994, entre otros) indica que los niños alrededor de los seis años han alcanzado el desarrollo cognitivo y lingüístico para comprender estas diferencias y así reconocer usos del lenguaje como errores, mentiras, sarcasmos y metáforas.

Tal reconocimiento supone un avance importante en la posibilidad de pensar sobre el lenguaje y no solamente usarlo; en otras palabras, representa un desarrollo metalingüístico, particularmente metaléxico, más elaborado. Esta competencia es fundamental para reconocer y apreciar usos figurativos del lenguaje como metáforas (Goddard, 2004; Schecter \& Broughton, 1991), metáforas e ironías (De Groot et al., 1995; Nippold, 1998; Winner E Gardner, 1993), así como también proverbios y frases idiomáticas (Nippold, 1998). El desarrollo metalingüístico ha sido relacionado con la comprensión lectora (por ejemplo, Gombert, 1992; Nagy, 2007). Particularmente Nagy (2007) plantea que los aspectos metalingüísticos de la comprensión lectora no se limitan a la comprensión de los significados de las palabras, sino que también implican un control de la comprensión de la forma lingüística que caracteriza muchas "sutilezas de significado -estilo, sarcasmo, metáfora y estado de ánimo-”.

La relación entre el avance en la comprensión lectora y un aspecto particular del conocimiento metalingüístico: el entendimiento de que es posible que una palabra o frase pueda tener más de un significado, ha sido investigada con niños escolares en edades entre ocho y catorce años (Yuill, 2007, 2009; Zipke, 2007, 2008; Zipke, Ehri, $\varepsilon$ Cairns, 2009). Dicho desarrollo metaléxico en relación con contemplar múltiples significados es crucial para reconocer metáforas, porque hace posible diferenciar entre el significado básico y el significado contextual de la o las palabras usadas metafóricamente.

Otro aspecto importante para reconocer un uso metafórico es el conocimiento de los dominios conceptuales. Este conocimiento permite al usuario de la lengua darse cuenta de que el o los términos usados metafóricamente deben interpretarse en razón del dominio conceptual prevalente de la metáfora y no del dominio conceptual al que 
pertenece dicho término o términos. Por ejemplo, para reconocer como metáfora Dinamarca derribó el Tratado de Maastricht (Croft, 2002, p. 162) es necesario advertir que derribar pertenece al dominio guerra y que este contrasta con el de actividad política al que pertenece tratado.

Por esta vía, es razonable asumir que el avance en el conocimiento de los dominios conceptuales va de la mano con el desarrollo metaléxico, dado que en la medida en que los niños tienen más conocimiento conceptual tienen más elementos para pensar sobre los sentidos que puede tener un término en un contexto lingüístico específico y advertir la diferencia entre los dos dominios conceptuales. Los resultados de Schecter y Broughton (1991) mostraron esta relación: el grupo de niños con bajo desempeño en consciencia o conocimiento metaléxico mostraron también una comprensión limitada de los conceptos implicados en las metáforas que les fueron presentadas.

Las metáforas no ocurren en el vacío, sino en un contexto discursivo particular. Levorato y Cacciari (1995, 2002) señalan el uso de la información contextual como uno de los factores claves en relación con procesamiento de metáforas y de lenguaje en general. La importancia del contexto pone de relieve que lectores con baja comprensión estarían en desventaja, dado que se ha mostrado que en general extraen menos información del contexto para construir su explicación de lo leído (Cain E Oakhill, 2004; Cain, Oakhill, \& Lemmon, 2004; Cain $\&$ Towse, 2008).

Los estudios con niños han estado centrados en explorar la comprensión de metáforas en inglés, comparando grupos de edad y usando en muchos casos frases o historias cortas construidas para la investigación. El trabajo en reconocimiento metafórico presentado aquí es innovador, dado que emplea textos de literatura infantil en español y explora no solo el efecto de la edad sino también la influencia de la comprensión lectora. El estudio estuvo orientado por las siguientes preguntas de investigación:

1. ¿Cuál es la influencia del grado escolar en el reconocimiento explícito de metáforas presentes en textos de literatura infantil?
2. ¿Cuál es la influencia del nivel de comprensión lectora en el reconocimiento explícito de metáforas en textos de literatura infantil?

\section{Método}

Con el propósito de generar un contexto lo más cercano posible al que los niños tienen en el colegio, el presente estudio se enmarcó en un pequeño proyecto de lectura - el cual fue presentado así a los niños y a sus familias - y comprendió varias sesiones de lectura en profundidad de fragmentos de algunos textos de literatura infantil, que los niños tenían a disposición en sus bibliotecas escolares. El conjunto de sesiones incluyó actividades para el reconocimiento e interpretación de metáforas; la parte que se presenta aquí es la del reconocimiento.

La selección del rango de edad y la configuración de los grupos de los participantes fueron determinadas de acuerdo con dos factores. El primero, que los niños hubiesen avanzado en el proceso de aprendizaje de la decodificación para reducir la posibilidad de interferencia con la exigencia para la comprensión lectora en sí misma. Igualmente, tener en cuenta el cambio importante que ocurre en el desarrollo del lenguaje alrededor de los ocho y diez años, que se evidencia en el aprendizaje de nuevo y más avanzado vocabulario, en una comprensión de lenguaje figurativo más elaborada y la construcción de un mayor número conceptos abstractos (Nippold, 1998), así como en avances significativos en el desarrollo metaléxico entre niños de diez, doce y catorce años (Schecter $\mathcal{E}$ Broughton, 1991). Debido a lo anterior se configuraron dos grupos de niños, uno de cuarto grado (ocho a diez años) y otro sexto grado (doce y catorce años).

El nivel de comprensión lectora fue establecido a través del test ACL (Català, Català, Molina y Monclús, 2001). Una prueba que, no obstante haber sido desarrollada para el contexto español, es la más completa para hispanohablantes, de acuerdo con la literatura revisada. La prueba ACL incluye preguntas relacionadas con varias áreas del conocimiento (Lenguaje, Matemáticas, Ciencias Naturales y Sociales) y diferentes tipos de textos (narrativos, 
informativos, poéticos), que apuntan a explorar tanto la comprensión de información literal como aquella no tan evidente y cuyo análisis demanda del lector un trabajo mental más exigente. El ACL, uno diferente para cada grupo escolar, fue aplicado a 469 niños al terminar el año académico, dado que el objetivo fue identificar el nivel de comprensión lectora que tenían los niños al finalizar su grado escolar. Los dos formatos del ACL se aplicaron entonces para grado tercero y quinto de primaria, de tal forma que el estudio de reconocimiento de metáforas se realizó tres meses después, cuando los niños cursaban cuarto y sexto grado.

Dadas las particularidades del test, en relación con términos y contextos propios de España, se realizaron algunos ajustes solo cuando se consideró necesario. Por ejemplo, en nombres de comidas, títulos de películas, lugares, que aparecen en algunos de los textos que preceden las preguntas de comprensión. Se incluyeron sinónimos de algunas palabras (por ejemplo, se cambió pequeños por niños; magdalenas por pastelitos) de tal forma que los términos del test hiciesen parte de los usos comunes en Colombia. Igualmente se ajustaron dos contextos que antecedían las preguntas de comprensión. Uno relacionado con cartelera de cine y el otro con un espacio natural típico de España. Estos fueron reemplazados por información de la oferta local de cine y por la información y mapa de un espacio natural de una zona conocida para los niños participantes del estudio.

\section{Materiales}

El material lingüístico utilizado en este estudio fue desarrollado siguiendo una combinación de dos métodos manuales de identificación de usos metafóricos, MIV (Cameron, 2003, 2006) y MIP (Pragglejaz Group, 2007), de una muestra de veinte textos de literatura infantil disponibles en las bibliotecas escolares de las instituciones educativas a donde asistían los niños. Un total de diez fragmentos de la muestra de literatura fueron seleccionados para la tarea de reconocimiento de metáforas. A fin de determinar los textos finales se tuvo en cuenta la variedad en los tópicos, el potencial interés y disfrute para todo el grupo de niños, la claridad, la profundidad, pero a la vez la posibilidad de que fuesen comprensibles; todo ello también debido al tiempo con el que se contaba para la recolección de los datos. Los diez fragmentos usados en el estudio de reconocimiento en literatura infantil fueron ajustados, solamente cuando fue necesario, buscando acortarlos sin perder la coherencia y cuidando de que contasen con información contextual relevante para facilitar su comprensión. Dichos fragmentos incluyeron diferentes frases metafóricas (en la Tabla 1 se presentan algunos ejemplos).

\section{Muestra}

La muestra estuvo conformada por 98 participantes (48 niñas y 50 niños) nativos y residentes en Colombia, seleccionados teniendo

Tabla 1. Algunos ejemplos de las metáforas objeto de reconocimiento

\begin{tabular}{ll}
\hline \multicolumn{1}{c}{ Metáfora } & \multicolumn{1}{c}{ Fuente } \\
\hline David lucha contra sus lágrimas & Minka \\
iNuestra casa es una isla! & Autora: María Seidemann (Alemania) [Traducción: Ana Garralón, \\
Sus miradas son dagas piratas & $1997]$ \\
Los cocuyos, son reyes en la noche & El valle de los Cocuyos \\
Los cocuyos, hijos de las estrellas & Autora: Gloria Cecilia Díaz (Colombia), 1986 \\
El viento rugía & La gente pobre \\
La tempestad soplaba & Autor: Leon Tolstoi (En: La ratoncita-niña y otros cuentos) (Rusia), \\
& [Traducción: Luz Amorocho, 1997] \\
Tano paseó la mirada por su cuarto & Tano en la frontera del tiempo \\
Un rayo de sol avanzaba por la colcha & Autor: Fernando Alonso (España), 1989 \\
Con el silencio, florecían mejor los pensamientos & \\
El corazón que se le explotaba & Tocotoc. El cartero enamorado \\
Tocotoc tuvo una idea que le iluminó el rostro & Autor: Clarisa Ruiz (Colombia), 1998 \\
\hline
\end{tabular}

Fuente: elaboración propia. 
en cuenta su nivel de comprensión lectora según el puntaje en la prueba ACL. En total fueron conformados cuatro grupos, dos de cuarto grado y dos de sexto grado. El grupo de cuarto grado estuvo conformado por 39 niños (veinte con bajo nivel de comprensión lectora y diecinueve con alto nivel) con edad promedio de 9;3, y el grupo de sexto por 59 niños (26 con bajo nivel y 33 con nivel alto) con edad promedio de $11 ; 7$.
La Tabla 2 presenta los niveles de comprensión lectora (CL) de cada uno de los grados escolares. La diferencia entre los dos grupos en cada grado escolar fue significativa. En grado cuarto $(F(1,38)$ $=143.88, p<.000, r=0.89)$ y en sexto $(F(1,58)$ $=224.30 p<.000, r=0.76$ ), lo que sugiere que los dos grupos se ajustan al propósito de la investigación.

Tabla 2. Niveles de comprensión lectora de los grupos participantes

\begin{tabular}{ccc}
\hline Grado escolar & Nivel de comprensión lectora & $M(D E)$ \\
\hline \multirow{3}{*}{ Cuarto $(\mathrm{N}=39)$} & Bajo $(\mathrm{N}=20)$ & $4.15(1.04)$ \\
& Alto $(\mathrm{N}=19)$ & $16.05(2.07)$ \\
& Total & $9.95(6.24)$ \\
Sexto $(\mathrm{N}=59)$ & Bajo $(\mathrm{N}=26)$ & $9.62(3.37)$ \\
& Alto $(\mathrm{N}=33)$ & $22.67(3.28)$ \\
Nota: Media en porcentajes con desviación estándar $(D E)$ entre paréntesis \\
\hline
\end{tabular}

Fuente: elaboración propia.

\section{Diseño}

Con el propósito de minimizar la influencia que pudiera tener el desconocimiento de los significados de las palabras en la comprensión de la tarea de reconocimiento metafórico, se concibieron dos situaciones de lectura con los diez fragmentos de textos de literatura. En la primera, los niños subrayaron las palabras desconocidas y, con base en este resultado, se realizaron sesiones de discusión y aprendizaje de nuevos significados.

En la segunda, los niños leyeron de nuevo cada fragmento con la consigna de encontrar partes del texto (palabras o frases) en donde hubiese, a su juicio, un uso metafórico. Previamente los niños participaron en sesiones grupales en donde se les explicó al respecto (ver apartado de 'procedimiento'). En este artículo se reportan los resultados y el análisis de esta tarea de reconocimiento.

Para el análisis estadístico de los resultados fue utilizado un ANOVA factorial con dos factores inter-sujeto (comprensión lectora y grado escolar). La unidad de análisis fue la palabra subrayada que correspondiese a la usada metafóricamente, en cuyo caso se contó como reconocimiento. Fueron contados como correctos aquellos subrayados que coincidían con la palabra o término usado metafóricamente o los que incluían dicho término. En la Tabla 3 se muestran algunos ejemplos de subrayados correctos (el término usado metafóricamente aparece en cursiva).

Tabla 3. Algunos ejemplos de subrayados correctos

\begin{tabular}{cc}
\hline$\#$ & Enunciado metafórico \\
\hline 1 & David lucha contra las lágrimas \\
2 & iNuestra casa es una isla! \\
3 & Los cocuyos, $\underline{\text { son reyes en la noche }}$ \\
4 & El viento rugía \\
5 & Tano paseó la mirada por su cuarto \\
6 & Con el silencio, florecían mejor los pensamientos \\
7 & Tocotoc tuvo una idea que le iluminó el rostro \\
8 & María era un ángel \\
9 & María era un ángel \\
\hline
\end{tabular}

Fuente: elaboración propia. 
Dos revisores independientes y provistos de una copia maestra de todos los fragmentos con los usos metafóricos subrayados realizaron el chequeo de lo realizado por los participantes.

\section{Procedimiento}

La tarea de reconocimiento de metáforas se basó en un estudio realizado por Steen (2004). Fue presentada con ejemplos del mismo término, por ejemplo, imán o volar, usado en diferentes contextos lingüísticos. Para estos términos se tuvieron entonces las frases ayer hicimos un experimento con un imán y su sonrisa es un imán, el pájaro vuela y el tiempo vuela. Se usaron cuatro términos para un total de ocho frases (cuatro con un sentido más básico y cuatro con sentido metafórico). Las sesiones de explicación de la tarea apuntaron a que los niños identificaran la diferencia entre los significados de las palabras de acuerdo con el contexto de uso y a que tuvieran la mayor claridad posible acerca del ejercicio de búsqueda de usos metafóricos que iban a realizar. La consigna dada fue la siguiente: "Ahora cada uno recibirá uno por uno diez textos, cada uno en una página, y debe subrayar las palabras que expresen un significado diferente al que refiere el texto, tal como fue explicado con los ejemplos". Se enfatizó que esta tarea era bien diferente a la realizada previamente (subrayado de palabras desconocidas). Cada niño realizó individualmente su tarea en un mismo salón de clase con el resto de participantes de su grupo escolar y del grupo alto o bajo en comprensión lectora, según fuese el caso. El orden de entrega de los fragmentos fue aleatorio y se realizó en dos sesiones (cada una para la mitad de los textos) para cada uno de los cuatro grupos.

\section{Resultados}

En la Tabla 4 se muestran los resultados de la tarea de reconocimiento en textos de literatura infantil, incluyendo la media y la desviación estándar (DE) por grado escolar (GE) y nivel de comprensión lectora (CL).

En general el reconocimiento de metáforas fue bajo (18.5\%). Los estudiantes de cuarto y sexto grado reconocieron solamente el $10.6 \%$ y el $23.7 \%$, respectivamente. Esto parece indicar que se trata de una tarea compleja. Los hallazgos también muestran una considerable variación en el reconocimiento de metáforas entre los niños con el mismo nivel de CL (Tabla 5).

Tabla 4. Reconocimiento de metáforas en literatura según GE y CL (en \%)

\begin{tabular}{|c|c|c|}
\hline Grado escolar & Nivel comprensión lectora & Total \\
\hline \multirow{3}{*}{ Cuarto $\quad N=39$} & Bajo $N=20$ & $(7.2)$ \\
\hline & Alto $\mathrm{N}=19$ & $16.0(12.2)$ \\
\hline & Total & $10.6(11.2)$ \\
\hline \multirow{3}{*}{ Sexto $N=59$} & Bajo $N=26$ & $13.6(12.6)$ \\
\hline & Alto $\mathrm{N}=33$ & $31.6(25.1)$ \\
\hline & Total & $23.7(22.3)$ \\
\hline \multirow{3}{*}{$\begin{array}{c}\text { Cuarto + Sexto } \\
N=98\end{array}$} & Bajo $N=46$ & $10.1(11.3)$ \\
\hline & Alto $N=52$ & $25.9(22.5)$ \\
\hline & Total & $18.5(19.7)$ \\
\hline
\end{tabular}

Nota: la media se presenta en porcentajes y la desviación estándar entre paréntesis

Fuente: elaboración propia. 
Tabla 5. Diferencias en reconocimiento metafórico en niños con similar nivel de CL

\begin{tabular}{cccc}
\hline Grado Escolar & Nivel de CL & Puntaje CL & Rango reconocimiento (\%) \\
\hline Cuarto & Bajo & 5 & $0-7.9$ \\
Cuarto & Alto & 16 & $5.3-28.9$ \\
Sexto & Bajo & 7 & $0-26.3$ \\
Sexto & Alto & 28 & $28.9-65.8$ \\
\hline
\end{tabular}

Fuente: elaboración propia.

Los resultados basados en el análisis estadístico ANOVA factorial muestran lo siguiente:

- Un efecto significativo de la comprensión lectora $\left(F_{1}(1,94)=16.10, p<.001, r=.38\right.$; esto implica que en general, los niños con baja comprensión de lectura reconocieron un porcentaje menor de metáforas $(M=10.1, D E=11.3)$ que aquellos con alta comprensión lectora $(M=25.9, D E=22.5)$.

- Un efecto significativo del grado escolar $\left(F_{1}(1,94)=11.17, p<.001, r=0,33\right)$. Lo cual indica que, en promedio, el porcentaje de metáforas reconocidas por los niños de cuarto grado $(M=10.6, D E=11.2)$ fue menor que el de los estudiantes de sexto grado $(M=23.7, D E=22.3)$.

- La interacción entre el grado escolar y la comprensión lectora no fue significativa, lo que implica que el efecto de cada uno de estos factores es independiente del otro.

\section{Discusión}

Los resultados indican que el grado escolar y el nivel de comprensión lectora influyen en el reconocimiento de metáforas y que sus efectos son independientes. Como era de esperarse, el grupo de sexto grado tuvo un desempeño mejor que el de cuarto y esto claramente apunta a la relevancia de la escolaridad, que favorece el avance en el vocabulario, el aprendizaje de significados figurativos más complejos y de conceptos más abstractos; así como el desarrollo del conocimiento metalingüístico. Todos estos aspectos relevantes para el reconocimiento de metáforas.

El hallazgo en relación con la comprensión lectora y su importante influencia en el reconocimiento de metáforas, con algunos niños de cuarto grado con comprensión lectora alta exhibiendo incluso un mejor desempeño que varios de sexto grado con bajo nivel de comprensión lectora, es plausible, si bien no ha sido mostrado antes. Es razonable esperar que un nivel alto de comprensión de lectura se refleje en habilidades metalingüísticas más desarrolladas (Nagy, 2007; Yuill, 2007, 2009; Zipke, 2007, 2008; Zipke, et al., 2009) que permiten reflexionar mejor sobre los significados y usos del lenguaje y utilizar más apropiadamente la información contextual, lo que facilitaría reconocer más metáforas. Adicionalmente, los niños con un nivel alto de comprensión lectora adoptan un estándar más alto de coherencia del texto (Perfetti, Landi, E Oakhill, 2005; Snowling, Oakhill, Nation, \& Cain, 2009), lo que favorece encontrar y resolver las inconsistencias en el texto, en lugar de ignorarlas o no notarlas, incluyendo aquellas generadas por el lenguaje figurativo.

Un nivel de comprensión lectora alto estaría también relacionado con una mayor capacidad para adaptar el modo de lectura para cumplir los objetivos de lectura (Cain, 1999). Por ejemplo, echar un vistazo a un texto para responder una pregunta general, leer en profundidad para elaborar una reseña o para aprender sobre un tópico particular o como en el presente estudio, hacer una lectura orientada a encontrar usos metafóricos. También es probable que los niños con comprensión lectora alta tengan un conocimiento conceptual más avanzado que aquellos con comprensión lectora baja, lo que favorece la diferenciación entre dominios conceptuales.

Si bien los resultados han mostrado la relevancia de la comprensión lectora en el reconocimiento de metáforas, es necesario profundizar en el estudio de los diferentes conocimientos que configuran lo que se denomina 'comprensión lectora' para explicar las 
diferencias en el desempeño en reconocimiento, como las encontradas en niños con el mismo nivel de comprensión lectora.

Una investigación adicional es relevante para avanzar en el conocimiento de la influencia en el reconocimiento metafórico, de aspectos implicados en la comprensión lectora tales como el desarrollo metalingüístico, la elaboración de inferencias, el monitoreo de la comprensión y el conocimiento sobre el género discursivo.

\section{Referencias}

Bowdle, B. \& Gentner, D. (2005). The career of Metaphor. Psychological Review, 112(1), 193-216.

https://doi.org/10.1037/0033-295X.112.1.193

Cain, K. (1999). Ways of Reading: How Knowledge and Use of Strategies Are Related to Reading Comprehension. British Journal of Developmental Psychology, 17, 295-312.

https://doi.org/10.1348/026151099165285

Cain, K. \& Oakhill, J. (2004). Reading Comprehension Difficulties. In T. Nunes \& P. E. Bryant (Eds.), Handbook of children's literacy (pp. 313-338). Dordrecht, The Netherlands: Kluwer.

https://doi.org/10.1007/978-94-017-1731-1_18

Cain, K., Oakhill, J., \& Lemmon, K. (2004). Individual Differences in the Inference of Word Meanings from Context: The Influence of Reading Comprehension, Vocabulary Knowledge, and Memory Capacity. Journal of Educational Psychology, 96, 671-681.

Cain, K., \& Towse, A. S. (2008). To Get Hold of the Wrong End of the Stick: Reasons for Poor Idiom Understanding in Children with Reading Comprehension Difficulties. Journal of Speech, Language, and Hearing Research, 51, 1538-1549. https://doi.org/10.1044/1092-4388(2008/07-0269)

Cameron, L. (1996). Discourse Context and The Development of Metaphor in Children. Current Issues in Language and Society, 3, 49-64.

https://doi.org/10.1080/13520529609615452

Cameron, L. (2003). Metaphor in Educational Discourse. London/New York: Continuum.

Cameron, L. (2006). MetNet Group: The Metaphor Analysis Project. Recuperado de http:// creet.open. ac.uk/projects/metaphoranalysis/index.cfm
Català, G., Català, M., Molina, E., y Monclús, R. (2001). Evaluación de la comprensión lectora. Pruebas ACL $\left(1^{\circ}-6^{\circ}\right.$ de primaria). Barcelona: Graó.

Croft, W. (2002). The Role of Domains in the Interpretation of Metaphors and Metonymies. In R. Dirven \& R. Pörings (Eds.), Metaphor and Metonymy in Comparison and Contrast (pp. 161-205). Berlin/ New York: Mouton de Gruyter.

https://doi.org/10.1515/9783110219197.16

De Groot, A., Kaplan, J., Rosenblatt, S. D. E Winner, E. (1995). Understanding versus Discriminating Nonliteral Utterances: Evidence for a Dissociation. Metaphor and Symbolic Activity, 10(4), 255-273.

https://doi.org/10.1207/s15327868ms1004_2

Gentner, D. \& Bowdle, B.F. (2001). Convention, Form, And Figurative Language Processing. Metaphor and Symbol, 16(3E4), 223-247.

https://doi.org/10.1080/10926488.2001.9678896

Gentner, D. E Bowdle, B.F. (2008). Metaphor as StructureMapping. In R. Gibbs (Ed.), The Cambridge Handbook of Metaphor and Thought (pp. 109-128). Cambridge: Cambridge University Press.

https://doi.org/10.1017/CBO9780511816802.008

Gibbs, R. W., Jr. (1993). Process and Products in Making Sense of Tropes. In A. Ortony (Ed.), Metaphor and thought (Second ed., pp. 252-276). Cambridge: Cambridge University Press.

Gibbs, R. W., Jr. (1994). The Poetics of Mind: Figurative Thought, Language, And Understanding. Cambridge: Cambridge University Press.

Glucksberg, S. (2008). How Metaphors Create CategoriesQuickly. In R. Gibbs (Ed.), The Cambridge Handbook of Metaphor and Thought (pp. 67-83). Cambridge: Cambridge University Press.

https://doi.org/10.1017/CBO9780511816802.006

Goddard, C. (2004), The Ethnopragmatic and Semantics of 'Active Metaphors'. Journal of Pragmatics, 36(7), 1211-1230.

https://doi.org/10.1016/j.pragma.2003.10.011

Gombert, J. E. (1992). Metalinguistic Development. London: Harvester Wheatsheaf.

Graesser, A. C., Mio, J. E Millis, K. K. (1988). Metaphors in Persuasive Communication. In D, Meutsch \& R. Viehoff, (Eds.), Comprehension on Literary Discourse: Results and Problems of Interdisciplinary Approaches (pp. 131-154). Berlin: de Gruyter.

Lakoff, G. E Johnson, M. (1980). Metaphors we Live By. Chicago: Chicago University Press. 
Levorato, M. C. E Cacciari, C. (1995). The Effect of Different Tasks on the Comprehension and Production of Idioms in Children. Journal of Experimental Child Psychology, 60, 261-283.

https://doi.org/10.1006/jecp.1995.1041

Levorato, M. C. \& Cacciari, C. (2002). The Creation of New Figurative Expressions: Psycholinguistic Evidence in Italian Children, Adolescents and Adults. Journal of Child Language, 29, 127-150.

https://doi.org/10.1017/S0305000901004950

Nagy, W. (2007). Metalinguistic Awareness and the VocabularyComprehension Connection. In R. K. Wagner, Andrea F. Muse, \& Kendra R. Tannenbaum (Eds.), Vocabulary Acquisition: Implications for Reading Comprehension (pp. 52-77). New York: The Guilford Press.

Nippold, M. A. (1998). Later Language Development. The School-Age and Adolescent Years (Second ed.). Austin, TX: Pro-Ed.

Olson, D. R. (1988). Or What's a Metaphor for? Metaphor and Symbolic Activity, 3(4), 215-222.

https://doi.org/10.1207/s15327868ms0304_2

Perfetti, C.A., Landi, N., \& Oakhill, J., (2005). The Acquisition of Reading Comprehension Skill. In M. J. Snowling \& C. Hulme (Eds.), The Science of Reading: A Handbook (pp. 227-247). Oxford: Blackwell.

https://doi.org/10.1002/9780470757642.ch13

Pragglejaz Group. (2007). MIP: A Method for Identifying Metaphorically Used Words in Discourse. Metaphor and Symbol, 22(1), 1-39.

https://doi.org/10.1080/10926480709336752

Schecter, B. \& Broughton, J. (1991). Developmental Relationships Between Psychological Metaphors and Concepts of Life and Consciousness. Metaphor and Symbolic Activity, 6(2), 119-143.

https://doi.org/10.1207/s15327868ms0602_4

Snowling, M. J., Oakhill, J., Nation, K., \& Cain, K. (2009). Reading Comprehension: Nature, Assessment and Teaching. Booklet from ESRC Seminar Series 'Reading Comprehension: From Theory to Practice'. York: Centre for Reading and Language.

Steen, G. J. (1994). Understanding Metaphor in Literature: An Empirical Approach. London/New York: Longman.

Steen, G. J. (2004). Can Discourse Properties of Metaphor Affect Metaphor Recognition? Journal of Pragmatics, 36, 1295-1313.

https://doi.org/10.1016/j.pragma.2003.10.014
Steen, G. J. (2008). The Paradox of Metaphor: Why We Need a Three-Dimensional Model for Metaphor. Metaphor and Symbol, 23(4), 213-241.

https://doi.org/10.1080/10926480802426753

Steen, G. J. (2011). The Contemporary Theory of Metaphor: Now New and Improved! Review of Cognitive Linguistics, 9(1), 26-64.

https://doi.org/10.1075/rcl.9.1.03ste

Torrance, N. y Olson, D. R. (1994). El papel de la alfabetización en la comprensión de la interpretación (On the role of literacy in children's understanding of interpretation). Substratum, 2(4), 23-45.

Winner, E. E Gardner, H. (1993). Metaphor and Irony: Two Levels of Understanding. In A. Ortony (Ed.), Metaphor and Thought (Second ed., pp. 425-443). Cambridge: Cambridge University Press.

https://doi.org/10.1017/CBO9781139173865.021

Yuill, N. (2007). Visiting Joke City: How Can Talking About Jokes Foster Metalinguistic Awareness in Poor Comprehenders? In D. McNamara (Ed.). Reading Comprehension Strategies: Theories, Interventions and Technologies (pp. 325-345). New York: Lawrence Erlbaum Associates.

Yuill, N. (2009). The Relation Between Ambiguity Understanding and Metalinguistic Discussion of Joking in Good and Poor Comprehenders: Potential for Intervention and Possible Processes of Change. First Language, 29(1), 65-79.

Zipke, M. (2007). The Role of Metalinguistic Awareness in the Reading Comprehension of Sixth and Seventh Graders. Reading Psychology, 28(4), 375-396.

https://doi.org/10.1080/02702710701260615

Zipke, M. (2008). Teaching Metalinguistic Awareness and Reading Comprehension with Riddles. The Reading Teacher, 62(2), 128-137.

https://doi.org/10.1598/RT.62.2.4

Zipke, M., Ehri, L. C., \& Cairns, H. S. (2009). Using Semantic Ambiguity Instruction to Improve Third Graders' Metalinguistic Awareness and Reading Comprehension: An Experimental Study. Reading Research Quarterly, 44(3), 300-321.

https://doi.org/10.1598/RRQ.44.3.4 